\title{
DRIVER FATIGUE: IS SOMETHING MISSING?
}

\author{
J. Christopher Brill, P. A. Hancock, and Richard D. Gilson \\ University of Central Florida \\ Orlando, Florida USA \\ E-mail: jbrill@pegasus.cc.ucf.edu
}

\begin{abstract}
Summary: Drowsiness and fatigue are serious problems in all transportation systems. One persistent issue is the lack of an agreed definition of these respective energetic states. Here we review the theoretical approaches (cognitive versus physiological) framing the driver fatigue problem. Known contributing factors to drowsiness include sleep debt, circadian rhythm, and shift work. However, we also suggest that certain inherent physiological reactions engaged in responses to motion itself represent a previously unrecognized but significant source of fatigue. We confirm the impact of this factor through comparisons of studies that either have or have not included prolonged motion.
\end{abstract}

\section{INTRODUCTION}

The National Highway Traffic Safety Administration (NHTSA) has estimated that driver fatigue and sleepiness were involved in an average of 56,000 vehicle crashes per year in the United States in the mid-1990s, over 1,500 of which resulted in fatalities (Expert Panel on Driver Fatigue and Sleepiness, 1997). While there are numerous potential causes of driver fatigue, the factors cited vary considerably depending upon one's theoretical orientation. Some researchers have emphasized cognitive factors such as attention and workload (e.g., Hancock \& Verwey, 1997). Other investigators approach driver fatigue from a physiological perspective, focusing on matters such as circadian rhythm and shift work (e.g., Folkard, 1997; Lenné, Triggs, \& Redman, 1997). It is our contention that one of the crucial contributors to driver fatigue is much more fundamental. We suggest that it is the motion itself, associated with vehicular transportation, that act as a significant influence in inducing the feelings of drowsiness, lassitude, and fatigue, which prove so destructive in these unforgiving operational environments.

\section{DEFINING DRIVER FATIGUE}

The question of driver fatigue is a subset of the general problem of fatigue. Since the classic paper by Muscio (1921), researchers have persistently encountered problems with the definition of this state. Maycock (1997), for example, dealt with the definition problem by addressing only "sleepiness," which is the aspect of fatigue that is perhaps the easiest to define and grasp. Summala, Häkkänen, Mikkola, and Sinkkonen (1999) did not directly define fatigue, but they discussed symptoms of fatigue such as sleepiness. Feyer, Williamson, and Friswell (1997) described fatigue as "a major occupational hazard" (p. 541), and they employed measures thought to tap into various dimensions of fatigue, but they do not provide a clear definition of the fundamental concept. The question of definition is not an unrecognized problem. Various works have sought to define fatigue (in the driving context or otherwise) including, but not limited to, the works by Muscio (1921), Brown (1994), Hancock and Verwey (1997), Nilsson, Nelson, and 
Carlson (1997), and Job and Dalziel (2001). While the problem of definition remains unresolved at this time, we approach this issue through a brief elaboration of potentiating factors.

\section{FACTORS RELATED TO DRIVER FATIGUE}

\section{Time of Day}

Lenné, Triggs, and Redman (1997) investigated whether driving performance varies across time of day. Participants drove a virtual track using a fixed-base driving simulator while performing the secondary visual reaction time task in six sessions spanning the 24-hour day. The authors found that performance on the driving task was poorest at 0600 and 0200 hours, with an afternoon dip around 1400 hours (Lenné, Triggs, \& Redman, 1997). These results confirm the crucial importance of circadian phase on performance, which obviously extends to complex skills such as driving. Interestingly, Lenné and colleagues' results are not too different from those of a macro-analysis of driving accident data by Folkard (1997). He found a significant peak in accident rates around 0300 hours and hypothesized that this effect might be due to circadian influence. Maycock (1997) likewise found an afternoon peak in rates of sleep-related accidents. Thus, circadian rhythm provides an oscillating baseline of potential fatigue state against which all other factors must be framed.

\section{Sleep Debt}

Not surprisingly, loss of sleep is also implicated in driver fatigue, and consequently, reduced performance. Fell and Black (1997) investigated driver fatigue incidents in cities, and found that $57 \%$ of drivers who had a fatigue-related incident reported insufficient sleep on the night before the incident happened. According to Brown (1994), loss of sleep "exacerbates the effects of fatigue on driving... and interacts with circadian rhythmicity" (p. 310). Williamson, Feyer, and Friswell (1996) investigated the influence of work practices on fatigue among long distance truck drivers. They found that in addition to work regimen, pre-trip fatigue levels (loss of sleep before the trip) influenced overall fatigue levels. Clearly, we are building here a summary of prepotentiating factors and the degree to which the individual is subject to the differentiating deleterious influences of each of these effects. However, for fatigue in transportation, we believe one vital influence has been neglected, and it is to this we now turn.

\section{Motion as a Factor in Fatigue and Drowsiness}

Graybiel and Knepton (1976) observed a collection of responses to motion including marked drowsiness, which they termed "sopite syndrome" (from the Latin sopire, meaning "to put to sleep"). Sopite syndrome sufferers exhibit drowsiness despite adequate rest. They also demonstrate difficulty concentrating, irritability, apathy, feelings of detachment, disinclination for work, and sleep disturbances. Graybiel and Knepton (1976) proposed that sopite syndrome could be a sole manifestation of motion sickness, implying that it is a form of motion sickness that can occur without what might be considered the traditional symptomology of motion sickness (e.g., nausea, dizziness). It can occur after adaptation to sickening motion (Graybiel \& Knepton, 1976), in mild motion settings involving vehicular transportation (such automobiles, airplanes, boats, trains, etc.), or in settings involving apparent self-motion (i.e., vection), such as simulators and virtual environments (Lawson \& Mead, 1998). 
It might be tempting to discount sopite syndrome as simply boredom completely unrelated to motion. However, Graybiel, Clark, and Zarriello (1960) found that persons lacking a normally functioning labyrinth were immune to the effects of sopite syndrome, suggesting that it is a phenomenon rooted in the vestibular system. In addition, sopite syndrome can manifest itself in fully rested persons in situations in which drowsiness cannot be attributed to boredom. Further, sopite syndrome can occur in dynamic, stimulating environments. A pilot suspected to be particularly susceptible to sopite syndrome once had the opportunity to ride tandem in a Navy F18 jet, only to find he had difficulty staying awake half-way through the trip (Mead \& Lawson, 1997)!

\section{Measuring the Effects of Mild Motion}

Currently, the primary measure for assessing sopite syndrome is the mild motion questionnaire (MMQ; Lawson, Kass, Muth, Sommers, \& Guzy, 2001). The MMQ is a 39-item scale in which participants rate the extent to which they are experiencing symptoms of motion on 5-point Likert-type scales. The MMQ contains four factors, which were given names deemed most descriptive of the types of symptoms/states the clusters of items measured: relaxed-content, head-body, drowsy-fatigued, and amotivational-poor concentration. Future work is needed to validate this assessment instrument alongside actual exposures such as those seen in Williamson, Feyer, and Friswell's (1996) work. Williamson et al. (1996) measured subjective fatigue states using three visual analog scales (VASs) tapping into constructs similar to those assessed by the MMQ: fresh-tired, clear headed-muzzy headed, and very alert-very drowsy. At present, however, a direct comparison cannot be made between MMQ subscale scores and the VAS ratings reported by Williamson et al. (1996) because they only indicated the means of summated scores, meaning they combined all three VASs sub-scores into a single total.

\section{Implications of Motion Induced Drowsiness}

If sopite syndrome results from variation in the vestibular system, then it is an integral, unavoidable part of driving and indeed all forms of transportation. Introducing sopite syndrome as a potential factor is important as it means that additional variance in the driver fatigue construct may be explained. If driver fatigue is partially attributable to sopite syndrome, then its suppression can be an important component in developing driver fatigue countermeasures. In customizing a fatigue countermeasure system, entering a value denoting an individual's susceptibility to motion might aid in improving the predictive aspects of such capabilities. Potentially, medication or some form of vestibular desensitization therapy could be used to counteract the effects of sopite syndrome for persons who are particularly susceptible to the effects of motion, and of course, improving motion management technologies in all forms of transportation would prove a valuable control advantage.

Considering susceptibility to sopite syndrome might also be useful in improving safety practices in commercial trucking or other transportation systems. Drivers who are particularly susceptible to sopite syndrome might be allotted scheduling accommodations to allow them to operate more safely. However, one potential danger in using sopite syndrome susceptibility in the workplace is discrimination. Professional trucking companies could attempt to use sopite syndrome susceptibility as a job selection parameter. Whether such a procedure is a legitimate form of selection would become a standards issue. 


\section{DIRECTIONS FOR FUTURE RESEARCH}

There are a number of directions that could be pursued to investigate our postulated relationship between driver fatigue and sopite syndrome. Initially, it is necessary to establish the extent to which motion susceptibility predicts the prevalence of sopite-like symptoms among drivers. This is an important first step, since susceptibility to motion varies widely between individuals. To this end, it would be critical to establish whether individuals without a functional vestibular labyrinth develop driver fatigue/sopite syndrome in the same manner as persons with a functional labyrinth (see Graybiel, Clark, \& Zarriello, 1960).

Another line of research needs to be directed to explore the effects of motion on circadian rhythm. In Graybiel and Knepton's (1976) original study, they observed that several participants who were exposed to motion experienced sleep disturbances that were not readily attributable to sickness. This suggests a vestibular-circadian link, but the connection was not established unequivocally. However, Morin (2002) identified neurons projecting from the vestibular nuclear complex to the intergeniculate leaflet (IGL) and the suprachiasmatic nucleus (SCN) of the circadian rhythm system. This interconnectivity between the vestibular system and SCN suggests that afferent vestibular signals are communicated to the structure responsible for maintaining circadian rhythmicity. With this being the case, it is reasonable to hypothesize that vestibular signals might modulate circadian rhythmicity, which could explain why sleep disturbances are associated with exposure to motion (Graybiel \& Knepton, 1976). If circadian rhythm shifts occur due to exposure to motion, then this would be a crucial factor for fatigue but also an important fundamental finding since the neurological pathways for such a connection seem evident. Such circadian shifts could affect shift scheduling for professional drivers.

Another empirical approach might be through a longitudinal study to assess susceptibility to sopite syndrome among applicants to professional driving schools to determine if such susceptibility correlates with data such as use of stimulants while driving (e.g., caffeine), driving safety record, break patterns, and job turnover. The problem with this type of study is the potential misuse of these types of data as inappropriate employment selection measures.

\section{SUMMARY}

Here, we have developed an argument that the cues associated with motion itself can induce fatigue-like symptoms. Instead of focusing most frequently on factors that occur prior to driving, we recommend concentrating more on factors directly associated with driving. This is not to diminish the role that factors such as sleep debt and circadian rhythm play in driver fatigue. Nor do we intend to convey that driver fatigue and sopite are identical constructs merely with different names. Rather, the vestibular effects of driving deserve more scrutiny in the context of driver fatigue, especially given the apparent relationship we have proposed. This link opens vistas of important research to determine the commonalties and distinguishing characteristics between driver fatigue and sopite syndrome in an attempt to reduce the significant and damaging impact that fatigue induces in all transportation systems operations. 


\section{REFERENCES}

Brown, I. D. (1994). Driver fatigue. Human Factors, 36(2), 298-314.

Expert Panel on Driver Fatigue and Sleepiness (1997). Drowsy driving and automobile crashes. Retrieved April 3, 2002 from http://www.nhtsa.dot.gov.

Fell, D. L., \& Black, B. (1997). Driver fatigue in the city. Accident Analysis and Prevention, 29(4), 463-469.

Feyer, A. M., Williamson, A., \& Friswell, R. (1997). Balancing work and rest to combat driver fatigue: An investigation of two-up driving in Australia. Accident Analysis and Prevention, 29(4), 541-553.

Folkard, S. (1997). Black times: Temporal determinants of transportation safety. Accident Analysis and Prevention, 29(4), 417-430.

Graybiel, A., Clark, B., \& Zarriello, J. J. (1960). Observations on human subjects living in a "slow rotation room" for periods of two days. American Medical Association Archives of Neurology, 3, 55-73.

Graybiel, A., \& Knepton, J. (1976). Sopite syndrome: A sometimes sole manifestation of motion sickness. Aviation, Space, and Environmental Medicine, 47, 873-882.

Hancock, P. A., \& Verwey, W. B. (1997). Fatigue, workload and adaptive driver systems. Accident Analysis and Prevention, 29(4), 495-506.

Job, R. F., \& Dalziel, J. (2001). Defining fatigue as a condition of the organism and distinguishing it from habituation, adaptation, and boredom. In P. A. Hancock and P. A. Desmond (Eds.), Stress, workload, and fatigue (pp. 466-475). Mahwah, NJ: Erlbaum.

Lawson, B. D., Kass, S., Muth, E., Sommers, J., \& Guzy, L. (2001). Development of a scale to assess signs and symptoms of sopite syndrome in response to mild or nonsickening motion stimuli. Proceedings of the 72nd Annual Meeting of the Aerospace Medical Association, Reno, NV.

Lawson, B. D., \& Mead, A. M. (1998). The sopite syndrome revisited: Drowsiness and mood changes during real or apparent motion. Acta Astronautica, 43, 181-192.

Lenné, M. G., Triggs, T. J., \& Redman, J. R. (1997). Time of day variations in driving performance. Accident Analysis and Prevention, 29(4), 431-437.

Maycock, G. (1997). Sleepiness and driving: the experience of U.K. car drivers. Accident Analysis and Prevention, 29(4), 453-462.

Mead, A. M., \& Lawson, B. D. (1997). Sopite syndrome case report I: Motion-induced drowsiness and mood changes in an individual with no other motion sickness symptoms - a case of "pure" sopite syndrome? Aviation, Space, and Environmental Medicine, 68(7), 648.

Morin, L. P. (2002). Circadian and Vestibular System Relationships. Retrieved April 9, 2002 from http://www.nsbri.org/Research/2001-2003/SleepProj7.html.

Muscio, B. (1921). Is a fatigue test possible? British Journal of Psychology, 12, 31-46.

Nilsson, T., Nelson, T. M., \& Carlson, D. (1997). Development of fatigue symptoms during simulated driving. Accident Analysis and Prevention, 29(4), 479-488.

Summala, H., Häkkänen, H., Mikkola, T., \& Sinkkonen, J. (1999). Task effects on fatigue symptoms in overnight driving. Ergonomics, 42(6), 798-806.

Williamson, A. M., Feyer, A.M., \& Friswell, R. (1996). The impact of work practices on fatigue in long distance truck drivers. Accident Analysis and Prevention, 28(6), 709-719. 